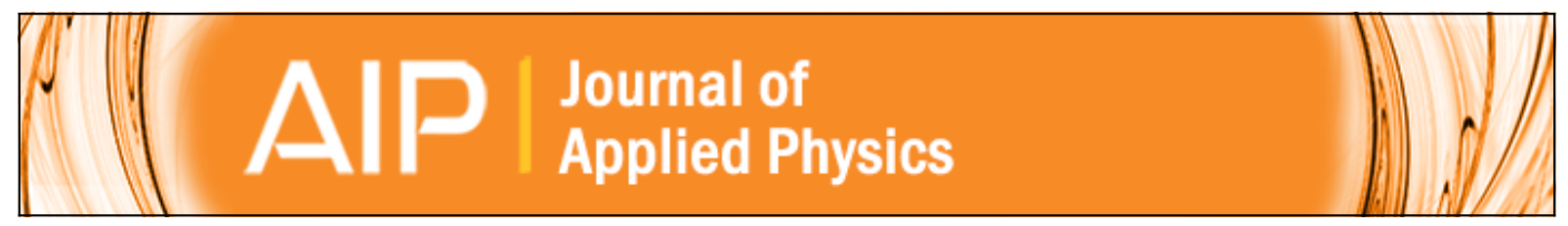

Relationship between the optical gap and the optical-absorption tail breadth in amorphous GaAs

J. H. Dias da Silva, R. R. Campomanes, D. M. G. Leite, Farida Orapunt, and Stephen K. O'Leary

Citation: Journal of Applied Physics 96, 7052 (2004); doi: 10.1063/1.1797541

View online: http://dx.doi.org/10.1063/1.1797541

View Table of Contents: http://scitation.aip.org/content/aip/journal/jap/96/12?ver=pdfcov

Published by the AIP Publishing

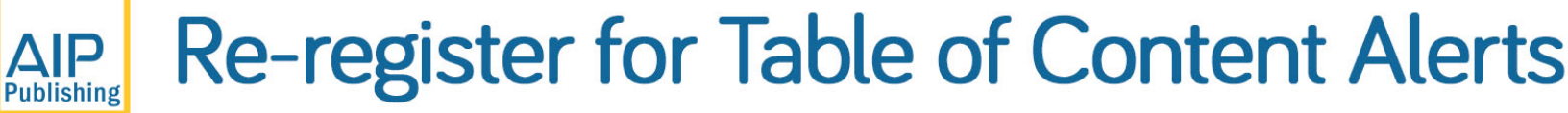

\section{Create a profile.




\title{
Relationship between the optical gap and the optical-absorption tail breadth in amorphous GaAs
}

\author{
J. H. Dias da Silva, R. R. Campomanes, and D. M. G. Leite \\ Departamento de Física, Faculdade de Ciências, Universidade Estadual Paulista, \\ CEP 17033-360 Bauru, São Paulo, Brazil \\ Farida Orapunt and Stephen K. O'Leary ${ }^{\text {a) }}$ \\ Faculty of Engineering, University of Regina, Regina, Saskatchewan S4S OA2, Canada
}

(Received 7 July 2004; accepted 3 August 2004)

\begin{abstract}
We study the relationship between the optical gap and the optical-absorption tail breadth for the case of amorphous gallium arsenide (a-GaAs). In particular, we analyze the optical-absorption spectra corresponding to some recently prepared a-GaAs samples. The optical gap and the optical-absorption tail breadth corresponding to each sample is determined. Plotting the optical gap as a function of the corresponding optical-absorption tail breadth, we note that a trend, similar to that found for the cases of the hydrogenated amorphous silicon and hydrogenated amorphous germanium, is also found for the case of a-GaAs. The impact of alloying on the optical-absorption spectrum associated with a-GaAs is also briefly examined. (C) 2004 American Institute of Physics.
\end{abstract}

[DOI: $10.1063 / 1.1797541]$

\section{INTRODUCTION}

Amorphous gallium arsenide (a-GaAs) is a promising electronic material that is used in a plethora of device applications. Thus far, this material has been used as an antiguide channel in vertical cavity surface-emitting lasers, ${ }^{1}$ as a buffer layer between silicon and gallium arsenide epitaxial layers, ${ }^{2}$ and as a host material for erbium doping. ${ }^{3}$ New applications for a-GaAs are being devised with each passing year. As with many other amorphous semiconductors, the optical properties of this material have been the focus of considerable investigation. ${ }^{4-8}$ From this research, key insights into the electronic character of a-GaAs have been gleaned.

The determination of the spectral dependence of the optical-absorption coefficient, $\alpha(\hbar \omega)$, has been a principal focus of studies into the optical response of a-GaAs for many years. In a crystalline semiconductor, the disorder present leads to a narrow tail in $\alpha(\hbar \omega)$, which encroaches into the otherwise empty gap region. In an amorphous semiconductor, however, there is a considerably greater level of disorder present, and the tail exhibited by $\alpha(\hbar \omega)$ is much broader. ${ }^{9}$ It is now widely held that the tail in the optical-absorption spectrum of an amorphous semiconductor is attributable to optical transitions involving tail states, these states encroaching into the otherwise empty gap region of an amorphous semiconductor. ${ }^{10-13}$ As the tail states arise as a result of the disorder inherent to the amorphous state, the breadth of the optical-absorption tail is often taken as a direct measure of the amount of disorder present in the material. ${ }^{14}$

In an amorphous semiconductor, such as a-GaAs, the disorder present has thermal and structural components. The thermal component reflects the thermal occupancy of the phonon states. The structural component, by a way of contrast, owes its existence to the structural disorder inherent to

\footnotetext{
${ }^{a)}$ Electronic mail: stephen.oleary @ uregina.ca
}

the amorphous semiconductor under consideration. In an effort to understand how these components individually contribute to the breadth of the optical-absorption tail associated with hydrogenated amorphous silicon (a-Si:H), Cody et al. ${ }^{14}$ performed an experimental investigation of their roles. In particular, structural disorder was deliberately introduced into a-Si:H films through the thermal evolution of hydrogen. A variety of measurement temperatures were employed in order to assess the role of thermal disorder. The data obtained from this study was consistent with the notion that the effect of the thermal and structural components of disorder are additive. The key result of this analysis, that the optical gap associated with a-Si:H decreases in response to the increases in the optical-absorption tail breadth, regardless of the exact nature of the disorder, suggests that disorder, rather than hydrogen content, determines the optical gap of a-Si:H.

Persans et al. ${ }^{15}$ performed a similar study for the case of the hydrogenated amorphous germanium (a-Ge:H). Rather than considering a-Ge: $\mathrm{H}$ as a homogeneous material, they instead envisioned it as a binary alloy, $\mathrm{Ge}_{1-x}(\mathrm{GeH})_{x}$, and provided experimental evidence to suggest that disorder related to alloying makes a significant contribution to the overall structural disorder present within this material; Cody et al. ${ }^{14}$ did not find compelling evidence suggesting disorder related to alloying present within a-Si:H, probably as a result of the fact that the hydrogen contents considered in the study of Cody et al. ${ }^{14}$ were too small. In a compound amorphous semiconductor, such as a-GaAs, which is fundamentally an alloy, i.e., a- $\mathrm{Ga}_{1-x} \mathrm{As}_{x}$, where $x$ denotes the alloy content, the disorder related to alloying is expected to make a potentially even more significant contribution to the overall structural disorder present.

In this paper, we study the relationship between the optical gap and the optical-absorption tail breadth for the case of a-GaAs. In particular, we analyze the optical-absorption 
spectra corresponding to some recently prepared a-GaAs samples. The optical gap and the optical-absorption tail breadth corresponding to each sample is determined. Then, plotting the optical gap as a function of the corresponding optical-absorption tail breadth, we will determine whether or not a trend, similar to that observed by Cody et al. ${ }^{14}$ for the case of a-Si:H and Persans et al. ${ }^{15}$ for the case of a-Ge:H, is also found for the case of a-GaAs. In an effort to assess the generality of these results, a comparison with other a-GaAs data from the literature is performed. We complete this analysis by considering the optical-absorption spectrum corresponding to a number of $\mathrm{a}-\mathrm{Ga}_{1-x} \mathrm{As}_{x}$ samples of varying alloy contents, with a view to developing an appreciation as to how the alloy content influences the optical absorption in this material. In order to provide a proper theoretical basis for the interpretation of these experimental results, the semiclassical optical-absorption analysis of O'Leary et al. ${ }^{11}$ is employed. From this analysis, insights into the optical response of a-GaAs are obtained.

This paper is organized in the following manner. In Sec. II, the experimental means, whereby the a-GaAs samples were prepared and their optical-absorption spectra were determined, is presented. Then, in Sec. III, we analyze the resultant optical-absorption spectra. A comparative analysis of a-GaAs and a-Si:H is also presented. The optical-absorption spectra corresponding to a number of a- $\mathrm{Ga}_{1-x} \mathrm{As}_{x}$ samples, with varying alloy contents, $x$, are then considered in Sec. IV, with a view to determining how the alloy content influences the optical absorption in this material. Finally, conclusions are drawn in Sec. V.

\section{FILM PREPARATION AND EXPERIMENT}

The a-GaAs films used to perform this study were deposited using the flash-evaporation technique on Corning 7059 glass substrates. The powder used for the depositions was obtained from high-purity small crystalline gallium arsenide (c-GaAs) pieces. The crucible temperature, $T_{\text {cruc }}$, was varied from sample to sample over the range from 1240 to $1900{ }^{\circ} \mathrm{C}$. The substrate temperature was kept constant at $25^{\circ} \mathrm{C}$ for all of the film depositions. ${ }^{16}$ The thickness of the resultant films, measured with a profilometer, was found to range from 400 to $1000 \mathrm{~nm}$. The composition of the films, determined using energy-dispersive $\mathrm{x}$-ray spectroscopy, suggested an excess of arsenic for all the samples prepared; it was found that $x=0.55 \pm 0.01$ for all of the a $-\mathrm{Ga}_{1-x} \mathrm{As}_{x}$ samples considered. Ex situ x-ray photoelectron measurements detected surface oxygen on the samples at the same concentration as on a reference c-GaAs wafer, i.e., 5 at. $\%$. We thus attribute the presence of this impurity to the surface oxidation of the air-exposed films. No other impurities were found within the detectability limit of the characterization techniques used. ${ }^{17}$ Further details on the preparation of these films, and on their characterization, are provided in the literature. ${ }^{7,8,18}$

Measurements of the transmittance and reflectance spectra of these a-GaAs films were obtained using a Lambda-9 Perkin-Elmer spectrophotometer in the visible and nearinfrared ranges. The corresponding optical-absorption spec-
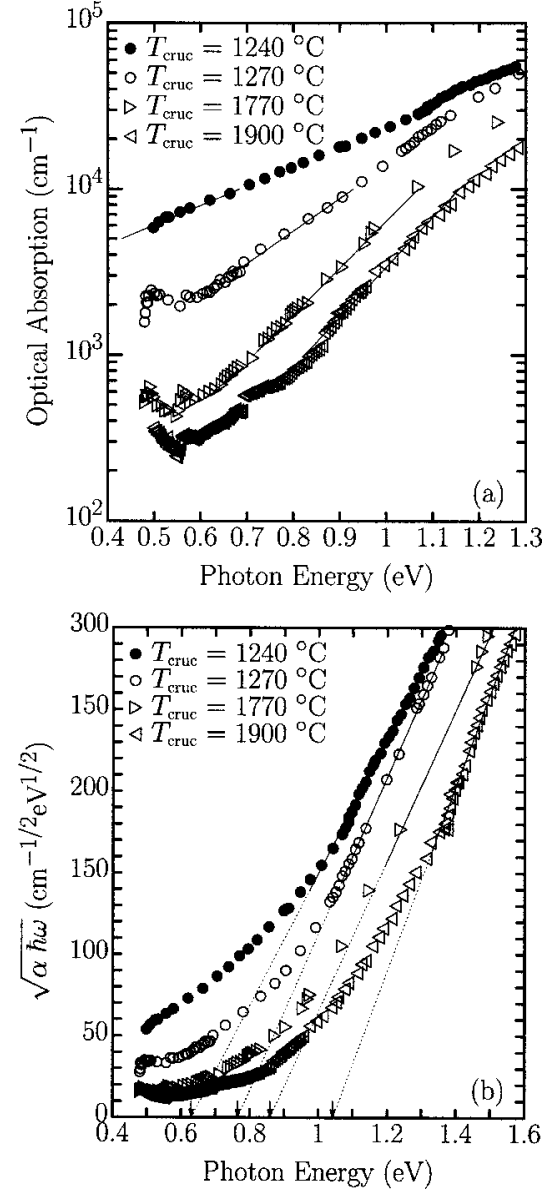

FIG. 1. (a) Optical-absorption spectra corresponding to four representative a-GaAs samples. The experimental data is depicted with the symbols. The fits of Eq. (1) are shown with the light solid lines. (b) The Tauc plots corresponding to the experimental data depicted in Fig. 1(a). The experimental data is depicted with the symbols. The fits of Eq. (2), and their extrapolations, are depicted with the solid and dotted lines, respectively. The Tauc optical gaps, determined from these extrapolations, are indicated with the small arrows.

tra were determined using standard thin-film optics techniques. ${ }^{19}$ For regions of the optical-absorption spectrum requiring a greater sensitivity, we employed photothermal deflection spectroscopy (PDS). The PDS results, which do not provide for an absolute determination of the opticalabsorption coefficient, were then calibrated with the results obtained from the transmittance and reflectance spectra. From this analysis, the optical-absorption spectrum corresponding to each a-GaAs sample was determined. Further details of our analysis are presented in the literature. ${ }^{7,8}$

\section{RESULTS AND ANALYSIS}

We analyzed a total of ten flash-evaporated a-GaAs samples for this analysis. In Fig. 1(a), we plot the opticalabsorption spectra corresponding to four of these samples. We note that there are significant variations in the opticalabsorption spectra. In particular, it is seen that the opticalabsorption spectrum corresponding to the sample prepared with $T_{\text {cruc }}=1240{ }^{\circ} \mathrm{C}$ is much broader, and encroaches into the gap region much deeper, than that prepared with $T_{\text {cruc }}$ $=1900{ }^{\circ} \mathrm{C}$. It is clear that the crucible temperature is playing 
TABLE I. The fitting parameters that arise from fitting Eqs. (1) and (2) to the optical-absorption spectra for the ten a-GaAs samples considered in this analysis. While we strove to fit Eq. (1) to the experimental data for $10^{3} \mathrm{~cm}^{-1} \leqslant \alpha(\hbar \omega) \leqslant 10^{4} \mathrm{~cm}^{-1}$ for all cases, for some of the data sets, we were forced to limit the range over which these fits were taken. The lower and the upper fit limits are specified in the table.

\begin{tabular}{ccccccc}
\hline \hline$T_{\text {cruc }}\left({ }^{\circ} \mathrm{C}\right)$ & $\alpha_{o}\left(\mathrm{~cm}^{-1}\right)$ & $E_{o}(\mathrm{meV})$ & Lower limit $\left(\mathrm{cm}^{-1}\right)$ & Upper limit $\left(\mathrm{cm}^{-1}\right)$ & $E_{g_{\text {Tauc }}}(\mathrm{eV})$ & $C_{\text {Tauc }}\left(\mathrm{cm}^{-1 / 2} \mathrm{eV}^{-1 / 2}\right)$ \\
\hline 1240 & $1.54 \times 10^{3}$ & 366 & $5 \times 10^{3}$ & $10^{4}$ & 0.630 & 405 \\
1270 & $1.48 \times 10^{2}$ & 220 & $3 \times 10^{3}$ & $10^{4}$ & 0.765 & 479 \\
1300 & $2.50 \times 10^{1}$ & 178 & $2 \times 10^{3}$ & $10^{4}$ & 0.809 & 411 \\
1350 & $9.93 \times 10^{-1}$ & 119 & $10^{3}$ & $10^{4}$ & 0.923 & 522 \\
1375 & $5.55 \times 10^{0}$ & 144 & $10^{3}$ & $10^{4}$ & 0.866 & 472 \\
1440 & $1.18 \times 10^{1}$ & 162 & $2 \times 10^{3}$ & $10^{4}$ & 0.827 & 411 \\
1500 & $9.14 \times 10^{0}$ & 157 & $10^{3}$ & $10^{4}$ & 0.815 & 396 \\
1600 & $9.12 \times 10^{0}$ & 151 & $2 \times 10^{3}$ & $10^{4}$ & 0.850 & 465 \\
1770 & $9.60 \times 10^{0}$ & 154 & $10^{3}$ & $10^{4}$ & 0.859 & 461 \\
1900 & $3.27 \times 10^{0}$ & 144 & $10^{3}$ & $10^{4}$ & 1.042 & 566 \\
\hline \hline
\end{tabular}

an important role in determining the electronic character of the resultant a-GaAs. Rather than determining the exact nature of this role, for the purposes of this analysis, we instead use the crucible temperature as a means of modulating the amount of disorder present in the samples. Through a direct comparison with the other a-GaAs results, the generality of our results may be confirmed.

We now quantify the relationship between the optical gap and the optical-absorption tail breadth. In order to determine the breadth of the optical-absorption tail, we follow the usual procedure in this analysis and fit an exponential functional dependence,

$$
\alpha(\hbar \omega)=\alpha_{o} \exp \left(\frac{\hbar \omega}{E_{o}}\right),
$$

to our optical-absorption experimental data, where $\alpha_{o}$ is a pre-exponential constant and $E_{o}$ denotes the breadth of the optical-absorption tail. Contrary to the approach adopted by Cody et al. ${ }^{14}$ in their analysis of a-Si:H, we do not, a priori, seek an Urbach "focus". Rather, we fit, in a least-squares sense, Eq. (1) to our optical-absorption experimental data. We find that Eq. (1) may be fitted to our a-GaAs experimental results for $10^{3} \mathrm{~cm}^{-1} \leqslant \alpha(\hbar \omega) \leqslant 10^{4} \mathrm{~cm}^{-1}$. ${ }^{20}$ The fits corresponding to the four representative a-GaAs opticalabsorption spectra depicted in Fig. 1(a) are also shown in Fig. 1(a). In Table I, the fits of Eq. (1), corresponding to the ten a-GaAs samples considered in this analysis, are tabulated.

For the determination of the optical gap, we employ the "Tauc" approach. ${ }^{21}$ That is, we fit, in a least-squares sense,

$$
\sqrt{\alpha(\hbar \omega) \hbar \omega}=C_{\text {Tauc }}\left(\hbar \omega-E_{g_{\text {Tauc }}}\right) \text {, }
$$

to the relevant experimental data, where $C_{\text {Tauc }}$ denotes the slope of the Tauc extrapolation and $E_{g_{\text {Tauc }}}$ represents the corresponding Tauc optical gap. The quantitative evaluation of $C_{\text {Tauc }}$ and $E_{g_{\text {Tauc }}}$ depend critically upon the range of data over which the fit of Eq. (2) is taken. For the purposes of this analysis, we perform our fit of Eq. (2) to the experimental data for which $2 \times 10^{4} \mathrm{~cm}^{-1} \leqslant \alpha(\hbar \omega) \leqslant 6 \times 10^{4} \mathrm{~cm}^{-1}$. The Tauc fits, corresponding to the four representative a-GaAs optical-absorption spectra depicted in Fig. 1(a), are shown in Fig. 1(b). In Table I, the fits of Eq. (2), corresponding to the ten a-GaAs samples considered in this analysis, are tabulated.

In order to demonstrate that our experimental results are consistent with the other a-GaAs results presented in the literature, in Fig. 2(a), we contrast the optical-absorption spectra of the four a-GaAs samples considered in Figs. 1(a) and 1(b) with representative a-GaAs optical-absorption experimental data from Theye et al. ${ }^{4}$ and Manssor and Davis; ${ }^{22}$ Theye $e t a l .{ }^{4}$ prepared their samples through a flash evaporation, whereas Manssor and Davis ${ }^{22}$ prepared their samples through sputtering. Differences in the optical-absorption spectra are evident. In particular, we find that the opticalabsorption spectra corresponding to two of our a-GaAs samples, that prepared with $T_{\text {cruc }}=1240^{\circ} \mathrm{C}$ and that prepared with $T_{\text {cruc }}=1270^{\circ} \mathrm{C}$, exhibit much broader opticalabsorption tails than the samples of Theye et al. ${ }^{4}$ and Manssor and Davis. ${ }^{22}$ This suggests that the $T_{\text {cruc }}=1240^{\circ} \mathrm{C}$ and $T_{\text {cruc }}=1270{ }^{\circ} \mathrm{C}$ a-GaAs samples have substantially greater amounts of disorder than the other a-GaAs samples. Nevertheless, the basic trend for all of the a-GaAs opticalabsorption spectra depicted in Fig. 2(a) appear to be similar.

In Fig. 2(a), we also present the optical-absorption spectrum corresponding to crystalline gallium arsenide (c-GaAs). ${ }^{23}$ It is interesting to note that this spectrum forms a loose lower bound to all of the presented a-GaAs opticalabsorption experimental data. That is, for any given value of $\hbar \omega$, the optical-absorption spectrum corresponding to a-GaAs exceeds that associated with c-GaAs. Moreover, it is seen that as the disorder is decreased, i.e., as $E_{o}$ decreases, that the optical-absorption spectrum corresponding to a-GaAs appears to approach that corresponding to c-GaAs. One could speculate that as the disorderless limit is approached, i.e., as $E_{o} \rightarrow 0$, that the optical-absorption spectrum associated with a-GaAs approaches that associated with c-GaAs. This would suggest a smooth and a continuous transition in the optical-absorption spectrum of gallium arsenide, from disordered a-GaAs to ordered c-GaAs.

The situation for the case of a-Si:H, however, is quite different. In Fig. 2(b), we plot the optical-absorption spectra corresponding to four representative a-Si:H data sets from Cody et al. ${ }^{14}$ The optical-absorption spectra, corresponding to the four representative a-GaAs samples depicted in Figs. 

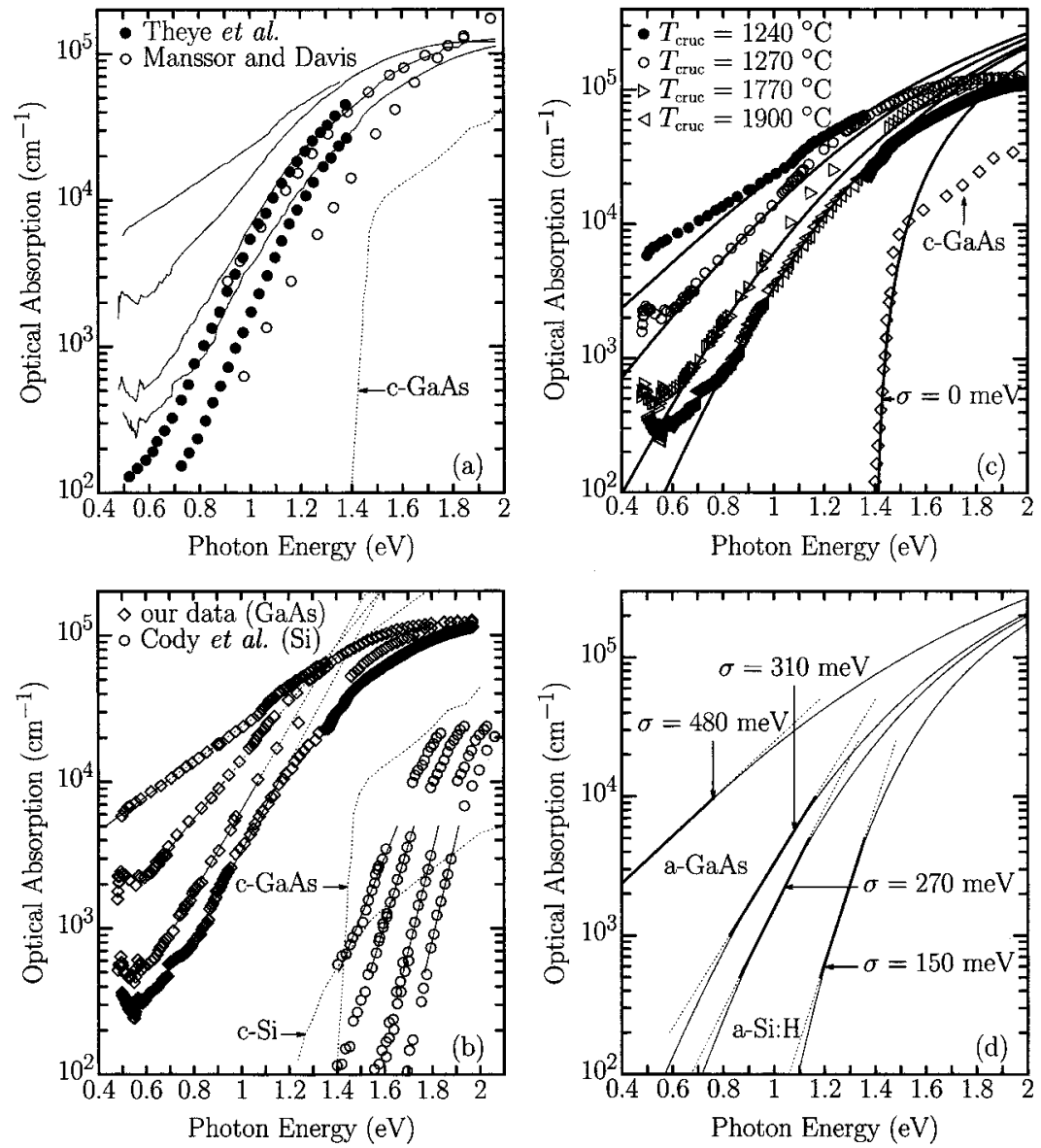

FIG. 2. (a) Optical-absorption spectrum of a-GaAs. The experimental data corresponding to the four representative a-GaAs samples depicted in Figs. 1(a) and 1(b) are shown with the light solid lines. The experimental data of Theye et al. (Ref. 4) and Manssor and Davis (Ref. 22) are depicted with the symbols. The optical-absorption spectrum corresponding to c-GaAs is depicted with the light dotted line (Ref. 23). (b) The optical-absorption spectra corresponding to a-GaAs and a-Si:H. The experimental data is depicted with the symbols: diamonds for the case of our a-GaAs experimental data and open circles for the a-Si:H experimental data of Cody et al. (Ref. 14). The fits of Eq. (1) are shown with the light solid lines. Extrapolations of these fits are shown with the light dotted lines. The optical-absorption spectra corresponding to c-GaAs and c-Si are clearly marked and indicated with the light dotted lines (Ref. 23). (c) The optical-absorption spectra corresponding to the semiclassical analysis of O'Leary et al. (Ref. 11) fit to the four representative a-GaAs samples depicted in Figs. 1(a) and 1(b). The experimental data is depicted with the symbols. The fits of the semiclassical analysis of O'Leary et al. (Ref. 11) to this experimental data are represented with the heavy solid lines. We find that we are able to fit the semiclassical analysis of O'Leary et al. (Ref. 11) to our a-GaAs optical-absorption data with $\sigma$ set to $310,350,420$, and $480 \mathrm{meV}$, and $E_{g_{0}}$ being set to $1.4 \mathrm{eV}$ for all cases, for the cases of $T_{\text {cruc }}$ set to $1900,1770,1270$, and $1240{ }^{\circ} \mathrm{C}$, respectively. The disorderless limit result, i.e., $\sigma=0 \mathrm{meV}$ and $E_{g_{o}}=1.4 \mathrm{eV}$, is also shown. The experimental c-GaAs results are shown with the open diamonds (Ref. 23). (d) Theoretical a-GaAs and a-Si:H optical-absorption results obtained using the semiclassical optical-absorption analysis of O'Leary et al. (Ref. 11). The results of the semiclassical optical-absorption analysis of O'Leary et al. (Ref. 11), corresponding to $\sigma$ set to $150,270,310$, and 480 meV, $E_{g_{o}}$ being set to $1.4 \mathrm{eV}$ for all cases, are shown with the light solid lines. The fits of Eq. (1), for $10^{3} \mathrm{~cm}^{-1} \leqslant \alpha(\hbar \omega) \leqslant 10^{4} \mathrm{~cm}^{-1}$ for the cases of $\sigma$ set to 310 and $480 \mathrm{meV}$, are shown with the heavy solid lines. The fits of Eq. (1), for $5 \times 10^{2} \mathrm{~cm}^{-1} \leqslant \alpha(\hbar \omega) \leqslant 5 \times 10^{3} \mathrm{~cm}^{-1}$ for the cases of $\sigma$ set to 150 and $270 \mathrm{meV}$, are shown with the heavy solid lines. Extrapolations of these fits, beyond the fitting ranges used, are depicted with the light dotted lines.

1(a), 1(b), and 2(a), are also shown, as are the opticalabsorption spectra corresponding to c-GaAs and crystalline silicon (c-Si). ${ }^{23}$ We note that as the disorder is decreased, i.e., as $E_{o}$ is decreased, that the optical-absorption spectrum corresponding to a-Si:H does not approach that corresponding to $\mathrm{c}-\mathrm{Si}$. This suggests that there is a fundamental discontinuity in the optical-absorption spectrum of silicon, from disordered a-Si:H to ordered c-Si. We opine that the discontinuity observed for the case of silicon in the disorderless limit, i.e., as $E_{0} \rightarrow 0$, fundamentally arises as a consequence of the indirect nature of the band structure associated with $\mathrm{c}-\mathrm{Si}$, the band structure associated with $\mathrm{c}-\mathrm{GaAs}$ being instead of a direct nature. Arguments in support of this point of view are presented by O'Leary. ${ }^{24}$

In order to develop a quantitative understanding as to how the disorder influences the optical-absorption spectrum associated with a-GaAs, we employ the semiclassical optical-absorption analysis of O'Leary et al. ${ }^{11}$ to interpret our optical-absorption experimental results. Noting that the spectral dependence of the optical-absorption coefficient, $\alpha(\hbar \omega)$, is, in large measure, determined by the spectral dependence of the joint density of states (JDOS) function, $J(\hbar \omega)$, the semiclassical analysis of O'Leary et al. ${ }^{11}$ focuses upon an evaluation of the JDOS function, $J(\hbar \omega)$. In this model, the overall form of the JDOS function is determined by averaging a local JDOS over a Gaussian distribution of local energy gaps. Two principal parameters characterize this model: (1) the mean energy gap, $E_{g_{o}}$, and (2) the energy-gap variance, $\sigma^{2}$, where $\sigma$ provides a measure of the amount of 
disorder. There is also a prefactor in the analysis, which is assumed to be a material constant for the case of a-GaAs.

In Fig. 2(c), we find that with selections of $\sigma$ between 310 and $480 \mathrm{meV}, E_{g_{o}}$ being set to $1.4 \mathrm{eV}$, and the prefactor being set to a constant for all cases, the semiclassical opticalabsorption analysis of O'Leary et al. ${ }^{11}$ is able to satisfactorily capture our a-GaAs experimental results over a broad range of photon energies; deviations from this semiclassical optical-absorption analysis arise as a result of defect absorption, the spectral dependence of the optical transition matrix element, ${ }^{25}$ and the deviations from square-root distributions of electronic states that occur in the band regions. ${ }^{26-28}$

The semiclassical optical-absorption analysis of O'Leary et $a{ }^{11}{ }^{11}$ provides us with an analytical tool, with which we can now quantitatively probe the disorderless limit. In particular, with all the semiclassical optical-absorption analysis parameters set as before, we study what happens as $\sigma$ is diminished to zero, this corresponding to the disorderless limit, i.e., $\sigma=0 \mathrm{meV}, E_{g_{o}}=1.4 \mathrm{eV}$, and the same a-GaAs prefactor. It is interesting to note that in this disorderless limit, the low optical-absorption semiclassical result essentially coincides with that of c-GaAs; deviations between the disorderless limit semiclassical result, i.e., $\sigma=0 \mathrm{meV}, E_{g_{o}}$ $=1.4 \mathrm{eV}$, and the same a-GaAs prefactor, and the opticalabsorption experimental c-GaAs result, which occur for values of optical absorption beyond $10^{4} \mathrm{~cm}^{-1}$, arise as a consequence of the stringent momentum conservation rules that shape the spectral dependence of $\alpha(\hbar \omega)$ for the case of c-GaAs, these conservation rules being relaxed for the case of a-GaAs. A similar result, corresponding to a different set of a-GaAs optical-absorption experimental data, was found previously by O'Leary. ${ }^{24}$

Recalling Fig. 2(b), it is interesting to note that the exponential region corresponding to the a-GaAs samples extends to higher optical-absorption values than that corresponding to a-Si:H. In particular, we find that the fit of Eq. (1) to the a-Si:H experimental data of Cody et al. ${ }^{14}$ is applicable for $5 \times 10^{2} \mathrm{~cm}^{-1} \leqslant \alpha(\hbar \omega) \leqslant 5 \times 10^{3} \mathrm{~cm}^{-1}$, i.e., this is a factor of 2 lower than that found for the case of a-GaAs. In order to understand this observation, we once again resort to the semiclassical optical-absorption analysis of O'Leary et al. ${ }^{11}$ In particular, in Fig. 2(d), we plot the optical-absorption results corresponding to a-GaAs, determined from the semiclassical optical-absorption analysis of O'Leary et al., ${ }^{11} \sigma$ being set to 310 and $480 \mathrm{meV}, E_{g_{o}}$ being set to $1.4 \mathrm{eV}$, and the prefactor being set as before for all cases, this corresponding to the range of $\sigma$ found in our a-GaAs analysis. We also plot the optical-absorption spectra corresponding to the semiclassical optical-absorption analysis of O'Leary et al., ${ }^{11}$ $\sigma$ being set to 150 and $270 \mathrm{meV}, E_{g_{o}}$ being set to $1.4 \mathrm{eV}$, and the prefactor being set as before for all cases, this corresponding to the range of $\sigma$ found in a previous a-Si:H analysis; ${ }^{24}$ we have selected a common $E_{g_{o}}, 1.4 \mathrm{eV}$, and a common prefactor value in order to facilitate a more direct comparison. It is noted that there is a distinctive curvature in these semiclassical optical-absorption spectra, which arises as a consequence of the Gaussian distribution of the local energy gaps. ${ }^{11}$ The presence of a curvature in the opticalabsorption spectrum makes the determination of the optical- absorption tail breadth, $E_{o}$, ambiguous, as the exact range of $\alpha(\hbar \omega)$, over which the fit of Eq. (1) to the experimental data should be taken, remains debatable. ${ }^{29,30}$ We note that with a broader optical-absorption tail, i.e., a larger $\sigma$, a region of apparently linear-exponential dependence is observed at larger values of $\alpha(\hbar \omega)$. Specifically, while the least-squares fits of Eq. (1) to the a-GaAs theoretical results, i.e., $\sigma$ set to 310 and $480 \mathrm{meV}$ for $10^{3} \mathrm{~cm}^{-1} \leqslant \alpha(\hbar \omega) \leqslant 10^{4} \mathrm{~cm}^{-1}$, lead to exponential fits that are essentially indistinguishable from the exact theoretical result over a wide range of $\hbar \omega$, if the same kind of fit is performed to the a-Si:H semiclassical results, i.e., $\sigma$ set to 150 and $270 \mathrm{meV}$, the resultant fits depart quite significantly from the theoretical results. On the other hand, the least-squares fits of Eq. (1) to the a-Si:H theoretical results, i.e., $\sigma$ set to 150 and $270 \mathrm{meV}$ for $5 \times 10^{2} \mathrm{~cm}^{-1}$ $\leqslant \alpha(\hbar \omega) \leqslant 5 \times 10^{3} \mathrm{~cm}^{-1}$, yield exponential fits that are essentially indistinguishable from the exact semiclassical result over a wide range of $\hbar \omega$. Thus, the fact that the fit of Eq. (1) occurs over a higher range of $\alpha(\hbar \omega)$ for the case of a-GaAs when contrasted with the case of a-Si:H simply arises as a consequence of the fact that the disorder in our a-GaAs samples is greater than that associated with the a-Si:H samples considered by Cody et al. ${ }^{14}$

The fits of Eq. (1), presented in Fig. 2(b), were performed without any assumption regarding the presence of an Urbach focus. In order to determine the applicability of the Urbach focus concept for the case of a-GaAs, in Fig. 2(b), we plot the extrapolations of the fits of Eq. (1) that were found. We note that these extrapolations do not all converge at a common point. In particular, it is seen that these fits intercept over a broad range of photon energies, between 1.2 and $1.6 \mathrm{eV}$, the corresponding optical-absorption values being between $10^{4}$ and $10^{6} \mathrm{~cm}^{-1}$. We thus conclude that the Urbach focus concept does not, strictly speaking, apply for the case of a-GaAs. ${ }^{31,32}$

In Fig. 3(a), we plot the optical gap as a function of the corresponding optical-absorption tail breadth for the a-GaAs samples we have considered in this analysis. While there are anomalies, in particular, the data point corresponding to the sample prepared with $T_{\text {cruc }}=1900{ }^{\circ} \mathrm{C}$, the overall trend is clear, i.e., the optical gap diminishes in response to the increases in the optical-absorption tail breadth. This result demonstrates that the trend observed by Cody et al. ${ }^{14}$ for the case of a-Si:H and Persans et al. ${ }^{15}$ for the case of a-Ge:H also occurs for the case of a-GaAs. A linear least-squares fit of this experimental data, excluding the $T_{\text {cruc }}=1900{ }^{\circ} \mathrm{C}$ anomalous point, yields

$$
E_{g_{\text {Tauc }}}=1.02-1.09 E_{o},
$$

where $E_{g_{\text {Tauc }}}$ and $E_{o}$ are in units of $\mathrm{eV}$, the error in $E_{g_{\text {Tauc }}}$ being of the order of $\pm 20 \mathrm{meV}$ in all cases. We note that, with the exception of the anomalous point, our experimental data lies very close to Eq. (3).

In order to demonstrate that the observed trend is not simply unique to our particular experimental data, in Fig. 3(a), we also include a-GaAs data obtained from other sources. In particular, results obtained from the experimental data of Theye et l. $^{4}$ and Manssor and Davis ${ }^{22}$ are included in Fig. 3(a). It is worth noting that while the data of Theye et 

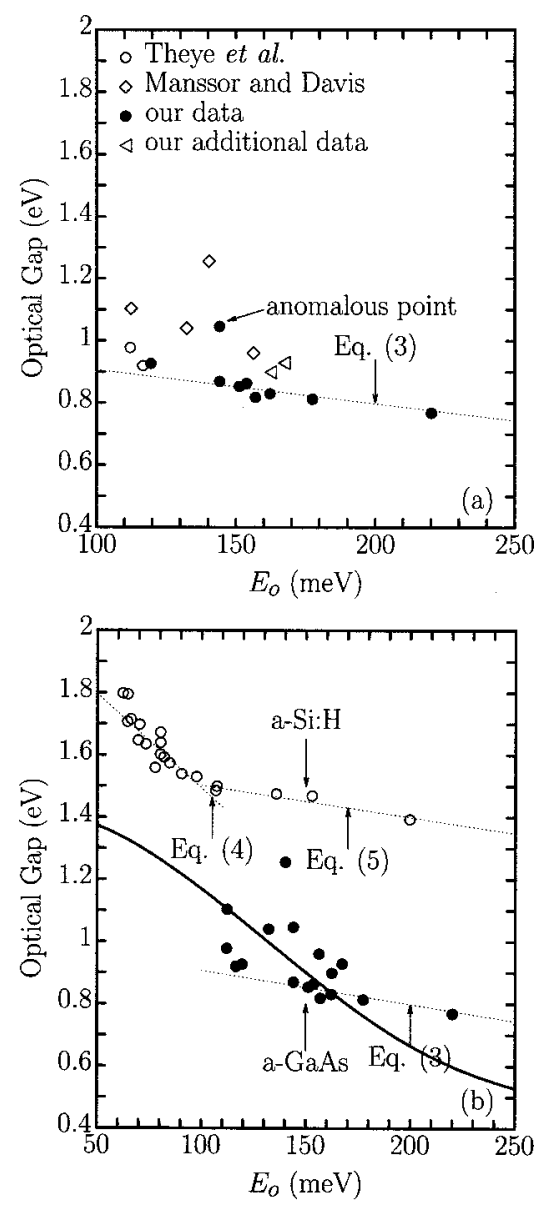

FIG. 3. (a) Optical gap of a-GaAs as a function of the optical-absorption tail breadth. All the a-GaAs experimental data is depicted with the symbols. The linear least-squares fit of our experimental data, i.e., Eq. (3), is depicted with the light dotted line. The additional data from our laboratory corresponds to flash-evaporated a-GaAs samples prepared with the substrate temperature held at $100{ }^{\circ} \mathrm{C}$. (b) The optical gap of a-GaAs and a-Si:H as a function of the optical-absorption tail breadth. The experimental a-GaAs data from us, Theye et al. (Ref. 4), and Manssor and Davis (Ref. 22), are included. The experimental a-Si:H data from Cody et al. (Ref. 14) and Viturro and Weiser (Ref. 33) are included. The a-GaAs experimental data is depicted with the solid points. The a-Si:H experimental data is depicted with the open points. The linear least-squares fits, i.e., Eqs. (3)-(5), are shown with the light dotted lines. The theoretical result, obtained using the semiclassical opticalabsorption analysis of O'Leary et al. (Ref. 11), is shown with the heavy solid line.

$a l .^{4}$ and our data correspond to unhydrogenated a-GaAs samples prepared through flash evaporation, the samples of Manssor and Davis ${ }^{22}$ correspond to intentionally hydrogenated a-GaAs samples prepared through radio-frequency sputtering. The data corresponding to two recently prepared flash-evaporated a-GaAs samples, with the substrate temperature held at $100{ }^{\circ} \mathrm{C}$, are also included; our other samples were prepared with the substrate temperature held at $25^{\circ} \mathrm{C}$. In order to allow for a fair comparison, we analyzed the other experimental data in exactly the same manner as was employed for the analysis of our own experimental data. We see that Eq. (3) forms a lower bound to this experimental data. We opine that the variations in the sample-preparation method are responsible for the observed deviations from Eq. (3), as suggested by the deviations from Eq. (3) in evidence for our samples prepared using the higher substrate temperature [see the open-triangle data points depicted in Fig. 3(a)].
We now perform a comparative analysis of a-GaAs and a-Si:H. For the case of a-Si:H, Fig. 3 of Cody et al. ${ }^{14}$ suggests that

$$
E_{g_{\text {Tauc }}}=2.10-6.07 E_{o},
$$

where $E_{g_{\text {Tauc }}}$ and $E_{o}$ are in units of eV. Eq. (4) has a form similar to that of Eq. (3). We do note, however, that the a-Si:H experimental data of Cody et al. ${ }^{14}$ exhibits considerably smaller values of $E_{o}$ than our a-GaAs experimental data. In order to allow for a fair comparison, we contrast all of the a-GaAs experimental data depicted in Fig. 3(a) with the a-Si:H experimental data of Cody et al. ${ }^{14}$ and Viturro and Weiser ${ }^{33}$ in Fig. 3(b), the optical-absorption tails exhibited by the a-Si:H experimental data of Viturro and Weiser ${ }^{33}$ being considerably broader than those of Cody et al. ${ }^{14}$ It is seen that the rate of decrease in the optical gap with the increased $E_{o}$ starts to decline for the higher values of $E_{o}$. This effect may well account for the differences in the slopes of Eqs. (3) and (4), i.e., the relationship between $E_{g_{\text {Tauc }}}$ and $E_{o}$ is fundamentally nonlinear, Eqs. (3) and (4) being applicable over different ranges of $E_{o}$. Focusing solely on the a-Si:H experimental data presented in Fig. 3(b) with $E_{o} \geqslant 100 \mathrm{meV}$, a linear least-squares fit analysis demonstrates that

$$
E_{g_{\text {Tauc }}}=1.60-1.02 E_{o},
$$

where $E_{g_{\text {Tauc }}}$ and $E_{o}$ are in units of $\mathrm{eV}$, the error being similar to that found for the case of Eq. (3). It is noted that the slopes of Eqs. (3) and (5) are, within the range of experimental error, essentially identical. We thus assert that the relationship between the optical gap and the optical-absorption tail breadth is similar in a-GaAs and a-Si:H.

Further insight into these results may be obtained through the use of the semiclassical optical-absorption analysis of O'Leary et al. ${ }^{11}$ In particular, using an approach similar to that outlined in Ref. 34 to determine the Tauc optical gap and the breadth of the optical-absorption tail, we plot the functional dependence of the Tauc optical gap on the opticalabsorption tail breadth. In order to allow for a fair comparison with the experimental results, we performed the determination of the Tauc optical gaps and the optical-absorption tail breadths in exactly the same manner as that employed for our experimental analysis. It is seen that the resultant dependence, depicted in Figure 3(b) with the heavy solid line, exhibits a functional form that captures the essence of the a-GaAs experimental results. We also note that as the disorderless limit is approached, i.e., as $E_{o} \rightarrow 0$, that the Tauc optical gap approaches the disorderless energy gap, $E_{g_{o}}$. This further bolsters our previously mentioned disorderless limit assertion.

\section{THE ROLE OF ALLOYING}

We now study how the alloy content, $x$, plays a role in influencing the optical response of $\mathrm{a}-\mathrm{Ga}_{1-x} \mathrm{As}_{x}$. Five a- $\mathrm{Ga}_{1-x} \mathrm{As}_{x}$ samples were prepared for the purposes of this investigation, the alloy content, $x$, being varied from sample to sample. These samples were deposited using the same flash-evaporation technique described previously. To produce variations in the alloy content, two different types of input 

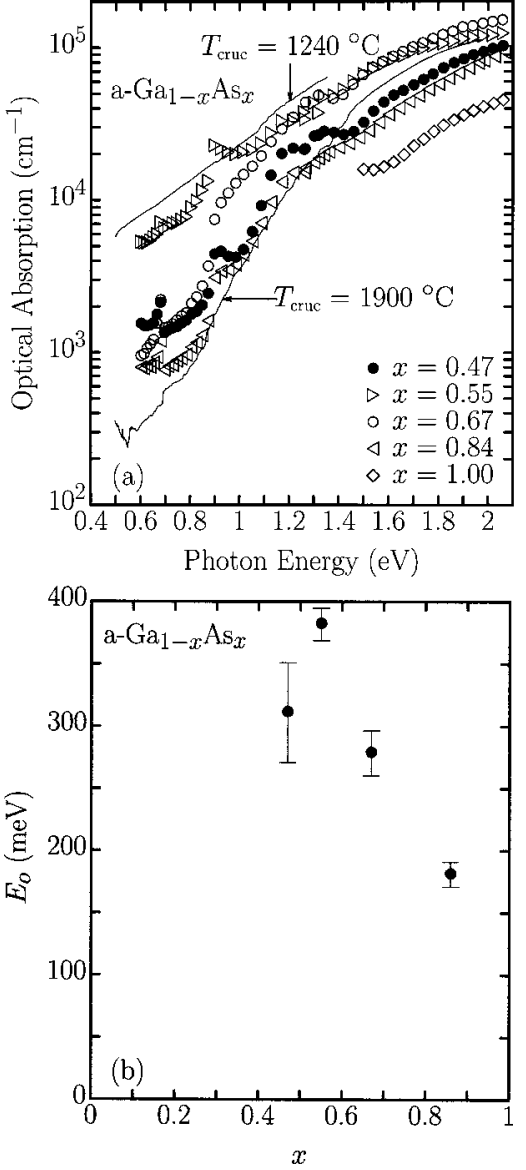

FIG. 4. (a) Optical-absorption spectra corresponding to five a- $\mathrm{Ga}_{1-x} \mathrm{As}_{x}$ samples of varying alloy contents. This experimental data is depicted with the symbols. The optical-absorption spectra corresponding to the $T_{\text {cruc }}=1240{ }^{\circ} \mathrm{C}$ and the $T_{\text {cruc }}=1900{ }^{\circ} \mathrm{C}$ a-GaAs results previously depicted are plotted with the light solid lines. (b) The dependence of the breadth of the optical-absorption tail, $E_{o}$, on the alloy content, $x$, for four of the a- $\mathrm{Ga}_{1-x} \mathrm{As}_{x}$ samples considered in Fig. 4(a). Error bars are shown.

material powders, namely, $6 \mathrm{~N}$ purity As powder and pcGaAs powder, were fed into the resistively heated tungsten crucible. For the flash-evaporation parameters used for these depositions, i.e., a crucible temperature of $1380{ }^{\circ} \mathrm{C}$ and a substrate temperature of $20^{\circ} \mathrm{C}$, the alloy content of the resultant films were found to be primarily determined by the input powder proportions used. The alloy contents found for the resultant $\mathrm{a}-\mathrm{Ga}_{1-x} \mathrm{As}_{x}$ films were 0.47, 0.55, 0.67, 0.84, and 1.00 .

In Fig. 4(a), we plot the spectral dependence of the optical-absorption spectrum corresponding to the five a $-\mathrm{Ga}_{1-x} \mathrm{As}_{x}$ samples considered in this analysis, these spectra being determined in exactly the same manner as specified previously, i.e., through measurements of the transmittance and the reflectance spectra and through PDS measurements. In order to provide for a basis of comparison, we also plot the spectral dependence of the optical-absorption coefficient corresponding to our $T_{\text {cruc }}=1240{ }^{\circ} \mathrm{C}$ and $T_{\text {cruc }}=1900{ }^{\circ} \mathrm{C}$ a-GaAs results, these results already being depicted in Figs. 1(a), 1(b), and 2(a)-2(c). We note that the spectral dependence of the optical-absorption coefficient associated with a $-\mathrm{Ga}_{1-x} \mathrm{As}_{x}$ is strongly influenced by the alloy content, $x$. In particular, it is seen that the optical gap and the breadth of the optical-absorption tail vary greatly with the alloy content, $x$. Plotting $E_{o}$ as a function of the alloy content, $x$, in Fig. 4(b), where $E_{o}$ is as determined previously, we find that $E_{o}$ varies considerably with $x$, exhibiting a peak in the neighborhood of $x \simeq 0.5$. Unfortunately, there are not enough data points, and the error associated with each such data point is too great, to draw definitive conclusions. Clearly, further research will be required in order to understand the exact role that the disorder related to alloying plays in contributing to the overall structural disorder present in a-GaAs.

\section{CONCLUSIONS}

In conclusion, we have studied the relationship between the optical gap and the optical-absorption tail breadth for the case of a-GaAs. By plotting the optical gap as a function of the corresponding optical-absorption tail breadth, we found that a trend, similar to that found for the cases of a-Si:H and $\mathrm{a}-\mathrm{Ge}: \mathrm{H}$, is also found for the case of a-GaAs. We conclude that the critical role played by the disorder in linking the optical gap with the breadth of the optical-absorption tail, observed in a-Si:H and a-Ge:H, also occurs for the case of a-GaAs, despite the disparate nature of these materials. Finally, the impact of alloying on the optical-absorption spectrum of a- $\mathrm{Ga}_{1-x} \mathrm{As}_{x}$ was briefly examined.

\section{ACKNOWLEDGMENTS}

The authors gratefully acknowledge financial support from FAPESP (Grant No. 97/06278-6). Two of the authors (F.O. and S.K.O.) acknowledge financial support from the Natural Sciences and Engineering Research Council of Canada. The use of equipment granted from the Canadian Foundation for Innovation, and equipment loaned from the Canadian Microelectronics Corporation, is acknowledged. Finally, the authors would like to thank the Photovoltaic Conversion Group at the University of Campinas for the use of their PDS facility.

${ }^{1}$ B.-S. Yoo, H. Y. Chu, H.-H. Park, H. G. Lee, and J. Lee, IEEE J. Quantum Electron. 33, 1794 (1997).

${ }^{2}$ K. I. Cho, W. K. Choo, J. Y. Lee, S. C. Park, and T. Nishinaga, J. Appl. Phys. 69, 237 (1991).

${ }^{3}$ A. R. Zanatta, Appl. Phys. Lett. 75, 3279 (1999).

${ }^{4}$ M. L. Theye, A. Gheorghiu, K. Driss-Khodja, and C. Boccara, J. NonCryst. Solids 77 \& 78, 1293 (1985).

${ }^{5}$ R. Murri, L. Schiavulli, N. Pinto, and T. Ligonzo, J. Non-Cryst. Solids 139, 60 (1992).

${ }^{6}$ S. H. Baker, S. C. Bayliss, S. J. Gurman, N. Elgun, J. S. Bates, and E. A. Davis, J. Phys.: Condens. Matter 5, 519 (1993).

${ }^{7}$ J. H. Dias da Silva and R. R. Campomanes, J. Non-Cryst. Solids 299-302, 328 (2002).

${ }^{8}$ R. R. Campomanes, J. Ugucione, and J. H. Dias da Silva, J. Non-Cryst. Solids 304, 259 (2002).

${ }^{9}$ G. D. Cody, in Hydrogenated Amorphous Silicon, Semiconductors and Semimetals Vol. 21B, edited by J. I. Pankove (Academic, New York, 1984), p. 11.

${ }^{10}$ S. K. O'Leary, S. Zukotynski, and J. M. Perz, Phys. Rev. B 51, 4143 (1995).

${ }^{11}$ S. K. O'Leary, S. Zukotynski, and J. M. Perz, Phys. Rev. B 52, 7795 (1995).

${ }^{12}$ S. K. O'Leary, S. R. Johnson, and P. K. Lim, J. Appl. Phys. 82, 3334 (1997).

${ }^{13}$ S. M. Malik and S. K. O'Leary, Appl. Phys. Lett. 80, 790 (2002).

${ }^{14}$ G. D. Cody, T. Tiedje, B. Abeles, B. Brooks, and Y. Goldstein, Phys. Rev. Lett. 47, 1480 (1981). 
${ }^{15}$ P. D. Persans, A. F. Ruppert, G. D. Cody, B. G. Brooks, and W. Lanford, AIP Conf. Proc. 120, 349 (1984).

${ }^{16}$ The substrate holder temperature was monitored during all the depositions using an externally fitted thermocouple. The thermocouple, situated at a distance of $3 \mathrm{~mm}$ from the substrate surface, was placed in a hole in the substrate holder from the external part of the chamber. Thermal contact of the thermocouple with the hole end was facilitated through the application of thermal grease. The thermocouple was encapsulated by a ceramic insulating tube so that only its tip reached the measurement point. We noted that the temperature of this point only changed by a few degrees during the depositions $\left(3{ }^{\circ} \mathrm{C}-8{ }^{\circ} \mathrm{C}\right)$. The fact that the depositions only lasted for 5 to 6 minutes coupled with the fact that the stainless-steel substrate holder is massive (approximately $2 \mathrm{~kg}$ ), and was connected to the chamber top flange (stainless steel) through a thermally conducting short neck, accounts for this observation.

${ }^{17}$ We can set an upper limit for the presence of hydrogen bonded to gallium or arsenic in the samples considered here as 0.1 at. \%. This limit was established by comparing the spectra of our samples with that of samples of similar thicknesses prepared through rf sputtering in the presence of hydrogen. In the hydrogenated samples, concentrations of less than 1 at. \% hydrogen correspond to the easily detectable spectral features, while in our samples the spectra were found to be completely featureless.

${ }^{18}$ R. R. Campomanes, J. H. Dias da Silva, J. Vilcarromero, and L. P. Cardoso, J. Non-Cryst. Solids 299-302, 788 (2002).
${ }^{19}$ R. Swanepoel, J. Phys. E 16, 1214 (1983).

${ }^{20}$ The a-GaAs experimental data does not span across this fitting range for all of the samples considered in this analysis. For these samples, the fits of Eq. (1) are performed with the available experimental data.

${ }^{21}$ J. Tauc, R. Grigorovici, and A. Vancu, Phys. Status Solidi 15, 627 (1966).

${ }^{22}$ M. I. Manssor and E. A. Davis, J. Phys.: Condens. Matter 2, 8063 (1990).

${ }^{23}$ S. M. Sze, Physics of Semiconductor Devices, 2nd ed. (Wiley, New York, 1981).

${ }^{24}$ S. K. O'Leary, Appl. Phys. Lett. 72, 1332 (1998).

${ }^{25}$ S. K. O'Leary, Appl. Phys. Lett. 82, 2784 (2003).

${ }^{26}$ W. B. Jackson, S. M. Kelso, C. C. Tsai, J. W. Allen, and S.-J. Oh, Phys. Rev. B 31, 5187 (1985).

${ }^{27}$ S. M. Malik and S. K. O'Leary, J. Non-Cryst. Solids 336, 64 (2004).

${ }^{28}$ S. M. Malik and S. K. O'Leary, submitted for publication.

${ }^{29}$ Such an ambiguity was commented upon by Roxlo et al. ${ }^{30}$ for the specific case of a-Si:H

${ }^{30}$ C. B. Roxlo, B. Abeles, C. R. Wronski, G. D. Cody, and T. Tiedje, Solid State Commun. 47, 985 (1983).

${ }^{31} \mathrm{~A}$ recent Urbach focus analysis for the case of a-Si:H has been presented by Orapunt and O'Leary (Ref. 32).

${ }^{32}$ F. Orapunt and S. K. O’Leary, Appl. Phys. Lett. 84, 523 (2004).

${ }^{33}$ R. E. Viturro and K. Weiser, Philos. Mag. B 53, 93 (1986).

${ }^{34}$ S. K. O'Leary, S. Zukotynski, and J. M. Perz, J. Non-Cryst. Solids 210, 249 (1997). 